\title{
Effective Performance of Location Aided Routing Protocol on Random Walk (RW) Mobility Model using Constant Bit Rate (CBR)
}

\author{
Ravi Kumar Verma \\ Student \\ SHIATS - Allahabad
}

\author{
Ashish Xavier Das \\ Assistant Professor \\ SHIATS - Allahabad
}

\author{
A.K. Jaiswal \\ H.O.D. (E\&C Department) \\ SHIATS - Allahabad
}

\begin{abstract}
MANET is the hoc network with collection of wireless nodes that communicate to each other without any infrastructure, access point or centralized. In mobile ad hoc network, each nodes have own capability to forwarding packet and receiving each other device. In this Article, showing the performance of location aided routing protocol LAR based on the broadcasting data on nodes placement scenario using Random walk point RW. Broadcast packet on CBR that means constant bit rate transfer constantly on Random way point model with Pause time is zero and showing the effective performance of location aided routing protocol in mobile ad hoc network. The analysis of performance is based on different network metrics such as number of hops counts, number of routes selected, number of RREP received, number of RREQ discarded for loop, number of RREQ received, number of duplicate RREQ received, number of RREQ retired, number of data packet dropped for no route, number of RREQ forward, number of RREQ TTL expired and Residual Battery Capacity using simulation tools Qualnet 6.0.
\end{abstract}

\section{Keywords}

MANET, LAR, RW, CBR, Qualnet 6.0.

\section{INTRODUCTION}

MANET is a collection of nodes in mobile, which dynamically form impermanent network communication without using any existing infrastructure, access point or central administration. Mobile network are quick and easy deployment of ad hoc network makes than feasible to use in military search and reuse operation meeting room and sensor network. Nodes can move randomly thus each nodes function as a router and forwarded packet to each other device in mobile ad hoc network. Mobility networks topology changes frequently. In wireless communication the wireless network plays important role. Now we are using wireless network in Industrial Application, Military Application and even in PAN network. Previously, difference between wireless and wired network is only in Communication Channel. Most common infrastructure based wireless network are cordless telephone, cellular network, Wi-fi, Microwave communication, Wi-Max, Satellite Communication and MANET.

Mobile network is defined as an autonomous system for wireless network of mobile nodes and associated host connected by wireless links. The Aim of this article is to perform LAR protocol showing effective performance compared to other routing protocol on Random walk point with pause time is zero second with the help of CBR using simulation tools Qualnet 6.0.

\subsection{Location Aided Routing}

The LAR is on demand routing protocol in which the overhead $f$ route discovery is decreased by utilizing location information of mobile nodes obtained using GPS. Location information is used to identity request zone and expected zone. Request Zone is a rectangular area that includes both sender and receiver.

\subsubsection{Expected Zone}

Expected Zone is the region where source node $\mathrm{S}$ thinks that the destination node $\mathrm{D}$ may contain at some time $t$ assuming that node $\mathrm{S}$ knows that the node $\mathrm{D}$ was at location $\mathrm{L}$ at time $t 0$ and current time is $t 1$.

\subsubsection{Request Zone}

The request zone is created as a rectangle with the source $\mathrm{s}$ in one corner and the expected zone in the opposite corner of the rectangle.

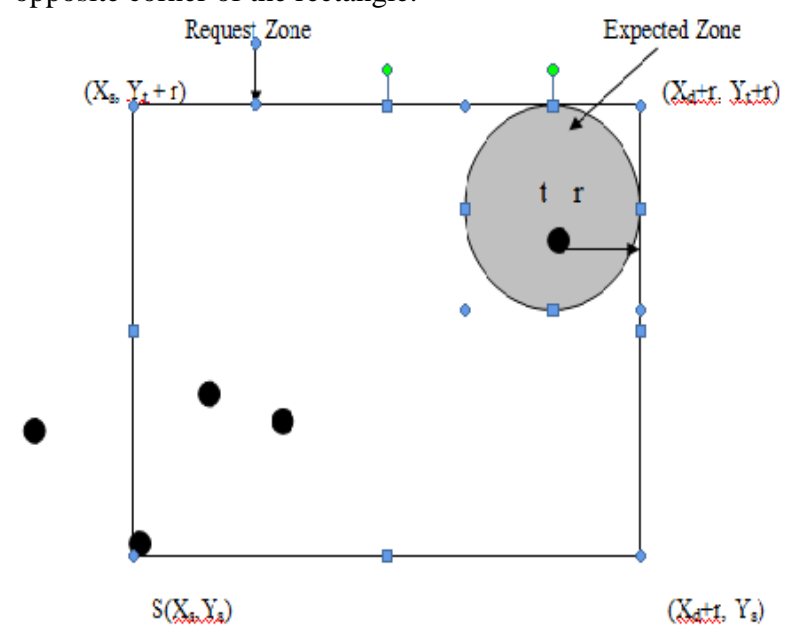

\subsection{Random Walk Mobility}

We simulate scenarios simulate on time of each scenario is $520 \mathrm{sec}$. All the simulations show the required results. Under simulation we check the behavior of LAR. In random walk mobility (RW) model with pause time is zero second, the nodes randomly selects a position, moves towards it in a straight line at a constant speed that is randomly selected from a range, and pauses at that destination. We get multiple graphs from simulations like first we get for delay, second is for the network load, and third one is for the throughput. Main goal of our simulation was to model the behavior of the routing protocols. We examined average statistics of the delay, network load and throughput for the MANET. A campus network was modeled within an area of $1500 \mathrm{~m} \mathrm{x}$ $1500 \mathrm{~m}$. The mobile nodes were spread within the area. We 
take the CBR traffic to analyze the effects on routing protocols. We configured the profile with CBR application. The nodes were wireless LAN mobile nodes with data rate of $11 \mathrm{Mbps}$. Random walk point mobility model was used in this simulation. The mobility model used is simple and it show more good mobility behavior. Mobile nodes move at a constant speed of $100 \mathrm{~m} / \mathrm{s}$, and when reaches the destination, the pause time is 0 (zero) sec and after that it choose a new random destination.

\subsection{CONSTANT BIT RATE (CBR)}

Constant Bit Rate (CBR) is a traffic generator. This UDPbased client-server application sends data from a client to a server at a constant bit rate. Random Waypoint mobility model in random waypoint mobility model, the nodes randomly selects a position, moves towards it in a straight line at a constant speed that is randomly selected from a range, and pauses at that destination. The node repeats this, throughout the simulation. In the simulation, Constant Bit-Rate (CBR) traffic flows are used with 4 packets/second and a packet size of 512 bytes.

\section{PARAMETERS FOR SIMULATION SETUP}

\begin{tabular}{|l|l|}
\hline Parameters & Values \\
\hline No. of Nodes & 25 \\
\hline Area & $1500 * 1500 \mathrm{~m}$ \\
\hline Routing Protocol & LAR \\
\hline Fading Model & Rayleigh \\
\hline Shadowing Model & Constant \\
\hline Energy Mode & Mica Motes \\
\hline Battery Model & Simple Linear Model \\
\hline Nodes Placement & RandomNodesPlacement \\
\hline Simulation Time & $520 \mathrm{sec}$ \\
\hline Channel Frequency & $2.4 \mathrm{GHz}$ \\
\hline Traffic Source & CBR \\
\hline Weather Mobility Interval & $100 \mathrm{~ms}$ \\
\hline Propagation Path loss Model & Two Way Model \\
\hline Mobility Pause Time & $0 \mathrm{sec}$ \\
\hline $\begin{array}{l}\text { BatteryChargeMonitoring } \\
\text { Interval }\end{array}$ & $60 \mathrm{sec}$ \\
\hline Battery Capacity & $1200 \mathrm{mAh}$ \\
\hline Propagation & $400 \mathrm{sec}$ \\
\hline Rate Packet Generation & $1 / \mathrm{sec}$ \\
\hline Mobility max-speed & $10 \mathrm{mps}$ \\
\hline Mobility min-speed & \\
\hline
\end{tabular}

\section{SIMULATION SCENARIOS FOR 25 NODES PLACEMENT}

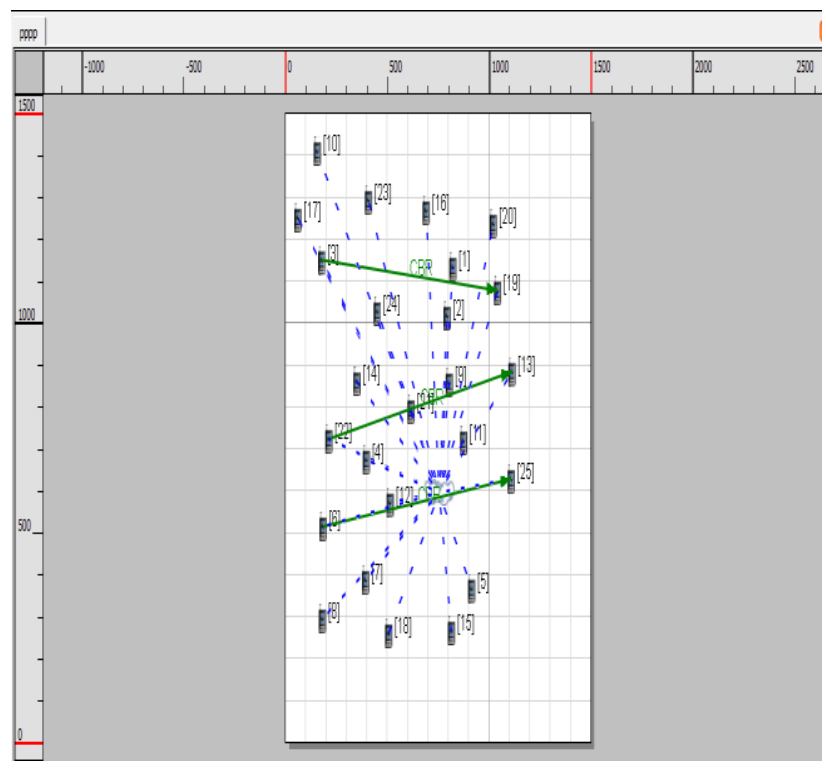

\subsection{PERFORMANCE METRIC}

1. Number of Hop Counts: Accumulated number of hops of all routes found by DSR at a node.

2. Number of Routes Selected: Total number of new routes found by a node.

3. Number of RREP Received: Total number of Route Replies received by a node.

4. Number of RREQ discarded for loop: Total number of Route Requests discarded for detecting a routing loop.

5. Number of RREQ received: Total number of Route Requests received by a node.

6. Number of Duplicate RREQ received: Total number of duplicate Route Requests received.

7. Number of RREQ Retried: Total number of Route Requests retransmitted by a node.

8. Number of RREQ Forwarded: Total number of packets dropped from DSR packet buffer because of no route.

9. Number of RREQ Forwarded: Total number of Route Requests forwarded by a node.

10. Number of RREQ TTL expired: Total number of Route Requests received by a node which was not relayed because of expiration of TTL.

11. Residual Battery Capacity: This model estimates the remaining service life of the battery at any time in the simulation. One important characteristic of the battery is that some amount of energy will be wasted when the battery is delivering the energy required by the circuit. 


\section{PERFORMANCE RESULT OF LOCATION AIDED ROUTING PROTOCOL VS NODES :-}

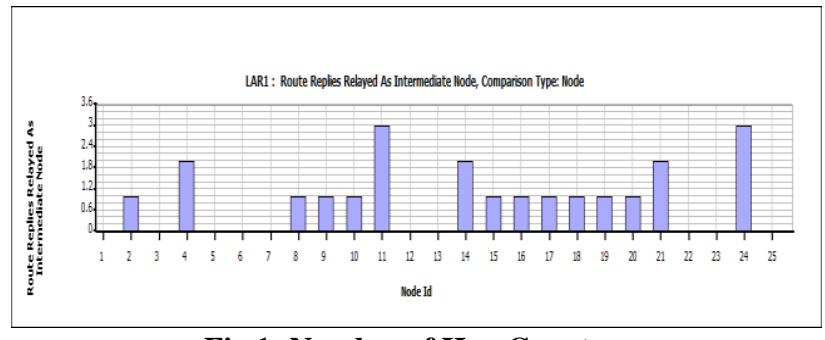

Fig 1: Number of Hop Counts

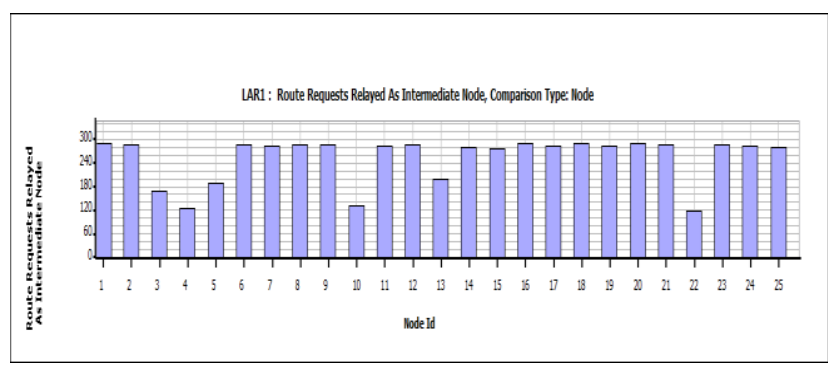

Fig 2: Number of Routes Selected

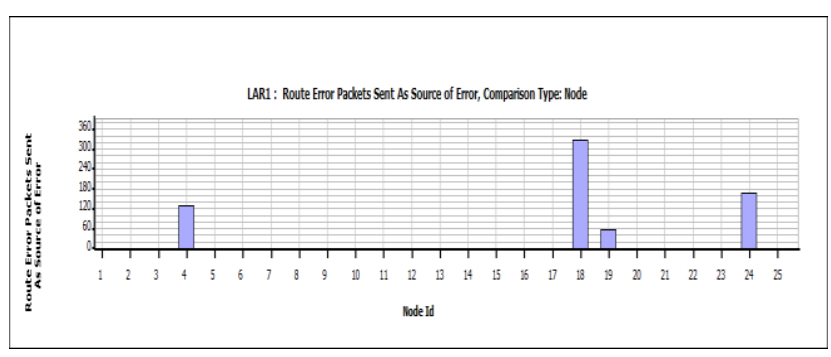

Fig 3: Number of RREP Received

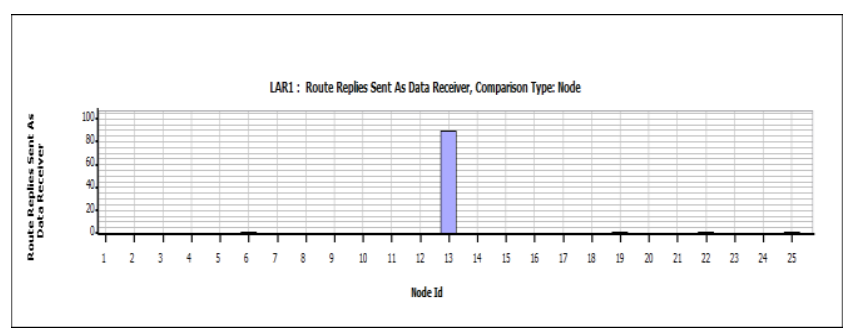

Fig 4: . Number of RREQ discarded for loop

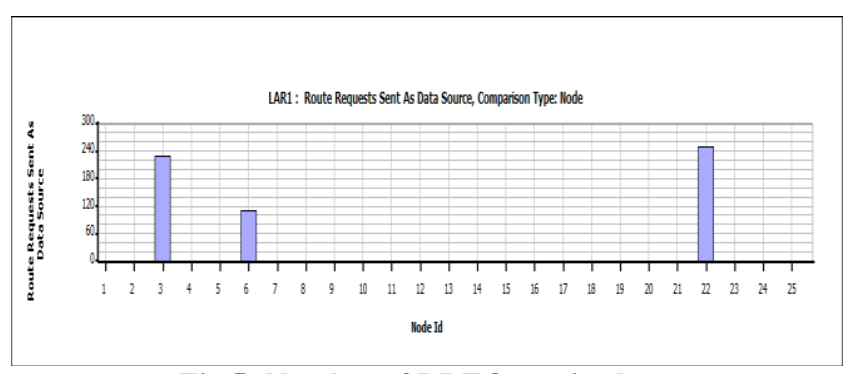

Fig 5: Number of RREQ received

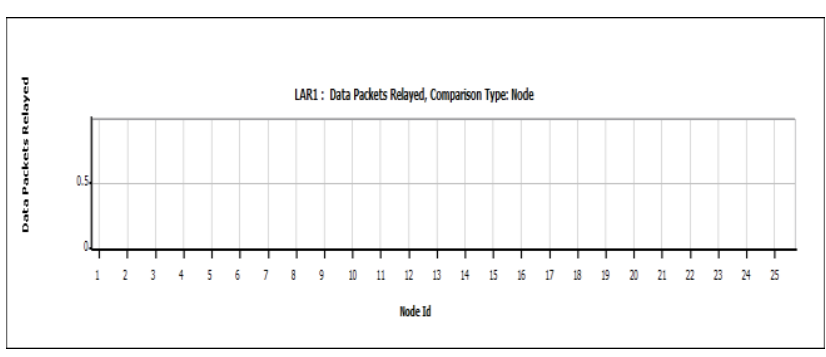

Fig 6: Number of Duplicate RREQ received

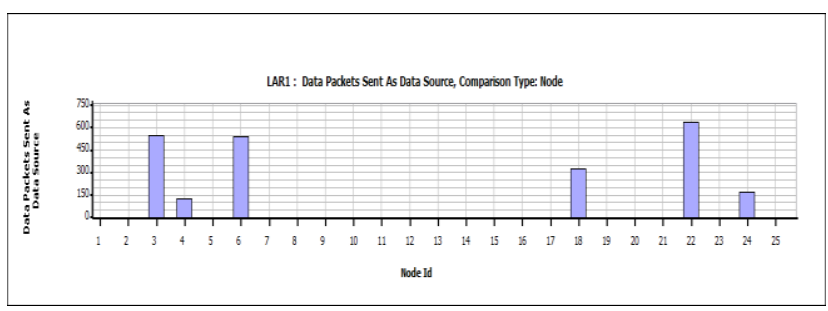

Fig 7: Number of RREQ Retried

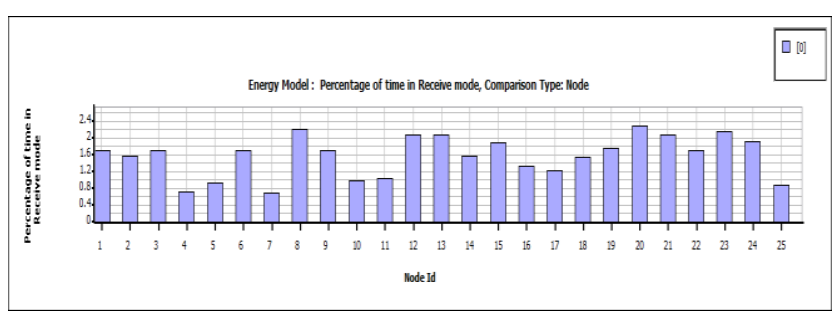

Fig 8: Number of RREQ Forwarded

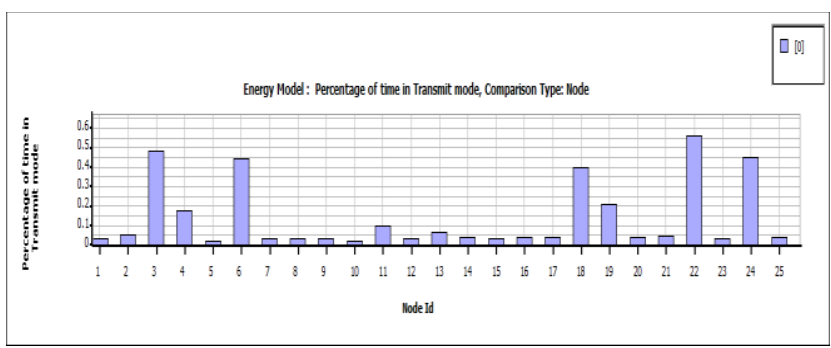

Fig 9: Number of RREQ Forwarded:

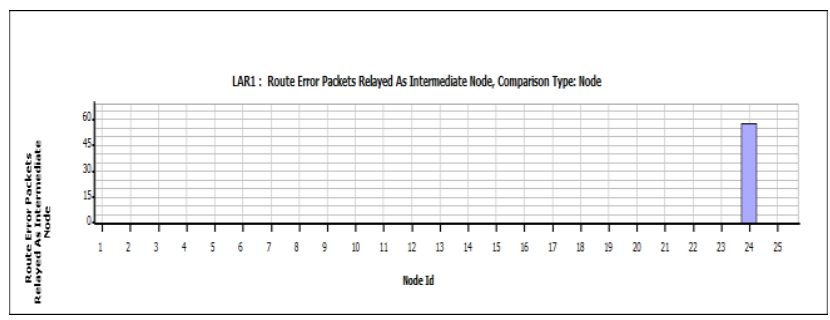

Fig 10: Number of RREQ TTL expired

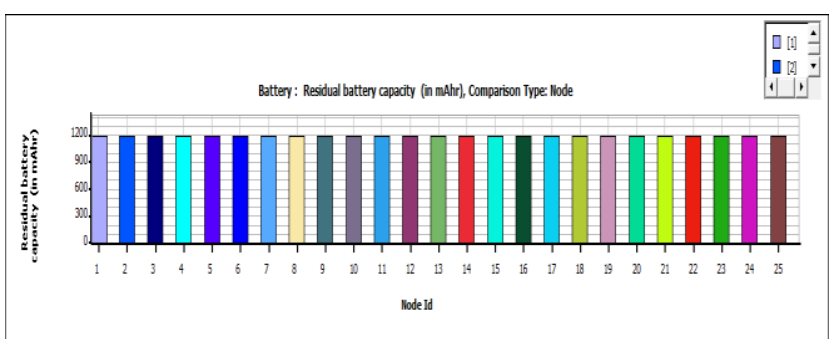

Fig 11: Residual Battery Capacity 


\section{CONCLUSION}

In this article showing the performance of Location Aided Routing (LAR) protocol using Qualnet 6.0 simulation tools as compared to other routing protocols on random walk-point model with pause time $=0$ second using constant bit rate (CBR). This article mainly gives effective performance on the performance metric such as Number of Hop Counts, Number of Routes Selected, Number of RREP Received, and Number of RREQ discarded for loop, Number of RREQ received, Number of Duplicate RREQ received, Number of RREQ Retried, and Number of Data Packets Dropped for no route, Number of RREQ Forwarded, Number of RREQ TTL expired and Residual Battery Capacity.

\section{REFERENCES}

[1] Shaily Mittal, Prabhjot Kaur, (2009), "PERFORMANCE COMPARISION OF AODV, DSR and ZRP ROUTING PROTOCOLS IN MANET'S".

[2] Parma Nand, Dr. S. C. Sharma (2011), "Routing Load Analysis of Broadcast based Reactive Routing Protocols AODV, DSR and DYMO for MANET".

[3] Sree Ranga Raju, Jitendranath Mungara, (2010), "Performance Evaluation of ZRP over AODV and DSR in Mobile Ad hoc Networks Using Qualnet".

[4] C.Siva Rammurty and B.S. Manoj (2011) , "Ad hoc wireless networks architectures and protocols".

[5] The Qualnet 6.0 simulator tools online available www.scalable-networks.com

[6] Draft-ietf-manet-dsr-07, "The Dynamic Source Routing Protocol for Mobile Ad Hoc Networks (DSR)".
[7] Naveen Choudhary (2011) "Constant Bit Rate Traffic Investigation for Network-on-Chip".

[8] Santosh Kumar S C Sharma Bhupendra Suman (2011), "Classification and Evaluation of Mobility Metrics for Mobility Model Movement Patterns in Mobile Ad-Hoc Networks".

[9] Supriya Agrahari and Suchismita Chinara (2003), "Simulation of Random Waypoint Mobility Model Using Colored Petri Nets".

[10] Dipankar Deb, Srijita Barman Roy, and Nabendu Chaki (2009), "LACBER: A NEW LOCATION AIDED ROUTING PROTOCOL FOR GPS SCARCE MANET".

[11] Young-Bae Ko and Nitin H. Vaidya,(2003), "LocationAided Routing (LAR) in mobile ad hoc networks".

[12] Tracy Camp Jeff Boleng Vanessa Davies,(2002) , "A Survey of Mobility Models for Ad Hoc Network Research".

[13] Anamika,(2012), “ Power Aware Location Aided Routing in Mobile Ad-hoc Networks".

[14] Mohammad A. Mikki (2009), "Energy Efficient Location Aided Routing Protocol for Wireless MANETs".

[15] Ruchi Aggarwal Amanpreet Kaur,(2009), “ Energy Efficient Zone Based Location Aided Routing Protocol for MANET",

[16] Suresh Kumar, Jogendra Kumar (2012), “Comparative Performance Study of Zone Routing Protocol over AODV and DSR Routing Protocols on Constant Bit Rate (CBR)". 